\title{
Predictores de Calidad de Vida en Pacientes con Diabetes Mellitus Tipo 1
}

\section{Predictors of Quality of Life in Patients with Type 1 Diabetes Mellitus}

\author{
Alberto Machado Romero \\ María Teresa Anarte Ortiz \\ María Soledad Ruiz de Adana Navas \\ Hptal. Punta de Europa de Algeciras(Cádiz) \\ Universidad de Málaga \\ Hptal. Universitario Carlos Haya (Málaga)
}

\begin{abstract}
Resumen. En el presente artículo se pretende estudiar si variables psicológicas (depresión, ansiedad-estado, ansiedad-rasgo y locus de control) y metabólicas (control glucémico e hipoglucemias graves) pueden predecir la calidad de vida de pacientes con diabetes mellitus tipo 1 (DM1). Treinta y tres pacientes con DM1 completaron una batería de tests psicológicos (DQOL, BDI, STAI y MHLC). Los resultados muestran que la depresión y la ansiedad-rasgo son buenos predictores de la calidad de vida de los pacientes con DM1. El resto de las variables (locus de control, hemoglobina glicosilada e hipoglucemias graves) no se relacionan con la calidad de vida. Estos resultados evidencian que las variables psicológicas (depresión y ansiedad-rasgo) pueden ser buenos predictores de calidad de vida en pacientes con DM1. Por tanto, según nuestros resultados, para mejorar la calidad de vida de las personas con esta enfermedad habría que promover la aplicación de tratamientos psicológicos dirigidos a reducir su sintomatología depresiva y ansiosa.
\end{abstract}

Palabras clave: calidad de vida, depresión, ansiedad, locus de control, control glucémico, diabetes mellitus tipo 1 .

\begin{abstract}
The aim of this article is to study whether psychological (depression, state-anxiety, trait-anxiety and locus of control) and metabolic variables (glucemic control and serious hypoglycemic events) can predict the quality of life in patients with type 1 diabetes mellitus (DM1). Thirty-three patients with DM1 completed a battery of psychological tests (DQOL, BDI, STAI and MHLC). The results show that depression and trait-anxiety are good predictors of quality of life in DM1 patients. The rest of the variables (locus of control, glycosylated hemoglobin and serious hypoglycemic events) are not related to the quality of life. These results demonstrate that psychological variables (depression and trait-anxiety) can be good predictors of quality of life in DM1 patients. Therefore, according to our results, to improve the quality of life of people with this disease it would be necessary to promote the application of psychological treatments directed at reducing their depressive and anxious symptomatology.

Key words: Quality of life, depression, anxiety, locus of control, glucemic control, type 1 diabetes mellitus.

La correspondencia sobre este artículo dirigirla a M. Teresa Anarte. Departamento de Personalidad, Evaluación y Tratamiento Psicológico. Facultad de Psicología. Universidad de Málaga. E-mail:anarte@uma.es

Agradecimientos: Este trabajo ha sido financiado por el Ministerio de Ciencia e Innovación (SEJ 2007-63786) y se ha realizado en colaboración con Unidad de Diabetes del Servicio de Endocrinología del Hospital Regional Universitario Carlos Haya de Málaga. Queremos expresar nuestro profundo agradecimiento a este excelente equipo, de reconocido prestigio científico, tan familiarizado con las tareas de investigación y, como cabeza visible, al Dr. Federico Soriguer Escofet, así como a los pacientes que aceptaron participar en este estudio.
\end{abstract}




\section{Introducción}

La Organización Mundial de la Salud (World Health Organization, 1994) define la calidad de vida como "la percepción del individuo de su posición en la vida en el contexto de la cultura y sistema de valores en los que vive y en relación con sus objetivos, expectativas, estándares y preocupaciones". Se trata de un concepto muy amplio que está influido de modo complejo por la salud física del sujeto, su estado psicológico, su nivel de independencia, sus relaciones sociales, así como su relación con los elementos esenciales de su entorno. De este modo, la calidad de vida es un constructo multidimensional que comprendería tres dimensiones fundamentalmente:

1. Dimensión física: percepción del estado físico o la salud, entendida como ausencia de enfermedad, los síntomas producidos por la enfermedad, y los efectos adversos del tratamiento.

2. Dimensión psicológica: percepción del individuo de su estado cognitivo y afectivo como el miedo, la ansiedad, la incomunicación, la pérdida de autoestima, la incertidumbre del futuro, etc. También incluye las creencias personales, espirituales y religiosas como el significado de la vida y la actitud ante el sufrimiento.

3. Dimensión social: percepción del individuo de las relaciones interpersonales y los roles sociales en la vida como la necesidad de apoyo familiar y social, la relación médico-paciente y el desempeño laboral.

Entre los diferentes autores existe cierto consenso en cuanto a que la evaluación de la calidad de vida debe hacerse tanto en términos objetivos, (necesidades del individuo, por ejemplo, salud, educación, etc.), como en términos subjetivos (percepción del individuo de lo que considera su nivel de bienestar). La calidad de vida subjetiva es un término que se superpone al de bienestar personal, subjetivo o psicológico (Fierro, 2006), definido, según Diener (1984), como "experiencia emocional placentera". Según Fierro (2006), el bienestar se asocia a una estabilidad emocional extravertida, ya que indicadores clínicos como la ansiedad y la depresión y el factor de personalidad "neuroticismo" correlacionan negativamente con el bienestar personal, mientras que el factor de personalidad "extraversión" correlaciona positivamente con el bienestar personal.

Los avances de la medicina han hecho posible aumentar notablemente la longevidad de las personas con enfermedad crónica, lo que no implica que haya mejorado necesariamente su calidad de vida. En este contexto nace el concepto "Calidad de Vida Relacionada con la Salud" (C.V.R.S.), en inglés "Health-Related Quality of Life" (H.R.Q.O.L). Numerosos trabajos de investigación científica emplean hoy este concepto, como una forma de referirse a "los distintos aspectos de la vida de una persona que están afectados firmemente por cambios en el estado de salud" (Schalock y Verdugo, 2003) o a "los efectos de la enfermedad y el tratamiento sobre el bienestar físico, emocional y social" (Cella et al., 1999). Esto refleja un cambio importante en las actitudes de los investigadores clínicos y de los servicios de salud en su elección de métodos para medir resultados. Las principales áreas investigadas en calidad de vida relacionada con la salud han sido cáncer, VIH, daño cerebral, esclerosis múltiple, enfermedades cardiovasculares, epilepsia, diabetes, asma, etc. (Schalock y Verdugo, 2003).

La investigación sobre la calidad de vida en pacientes con diabetes mellitus es bastante reciente. En una revisión realizada en adultos con ambos tipos de diabetes, Rubin y Peyrot (1999) afirman que la calidad de vida de estas personas es inferior a la de la población general, sobre todo en funcionamiento físico y bienestar. Se han descrito múltiples factores relacionados con la diabetes que pueden afectar la calidad de vida de los pacientes con esta enfermedad (Anarte, 2004):

- el propio impacto del diagnóstico.

- las demandas del tratamiento que pueden poner a la persona con diabetes o a sus familiares ante situaciones que, con frecuencia, producen la aparición de reacciones emocionales negativas.

- la incertidumbre sobre posibles complicaciones futuras.

- el miedo a las hipoglucemias por las consecuencias negativas de éstas (físicas, cognitivas, motoras, etc.).

- frustración ante niveles altos o bajos de glucemia inexplicables.

- el cumplimiento diario del régimen médico que 
impone restricciones en el estilo de vida familiar (inyecciones, análisis, planificación de comidas, ejercicio físico, etc.).

Según Rubin y Peyrot (1999), la calidad de vida de pacientes adultos con diabetes mellitus mantiene una relación muy consistente con las complicaciones de la enfermedad. Asimismo, se ha registrado una disminución de la calidad de vida de adultos con diabetes mellitus tipo 1 (DM1) a medida que aumenta el número y la severidad de los síntomas de las complicaciones médicas de la enfermedad (Aalto, Uutela y Aro, 1997; Hahl et al., 2002). Estos hallazgos también se han encontrado en una revisión realizada por Ambler y colaboradores (2006) en niños y adolescentes australianos con DM1.

Rubin y Peyrot (1999), tras revisar diversos estudios que han analizado la relación entre el control glucémico del paciente con diabetes mellitus y su calidad de vida, concluyen que un buen control glucémico se asocia con un aumento en la calidad de vida percibida, sobre todo, en aquellos estudios que evalúan la calidad de vida mediante instrumentos específicos de la diabetes más que generales. Estos resultados se han encontrado tanto en adultos (Tan, Shafiee, Wu y Rey, 2005; Weinger y Jacobson, 2001; Wikby, Hörnquist, Stenström y Andersson, 1993) como en niños y adolescentes con DM1 (Guttmann-Bauman, Flaherty, Strugger y McEvoy, 1998). Sin embargo, también existen estudios en los que no se ha relacionado la calidad de vida con la hemoglobina glicosilada en adolescentes con DM1 (Grey, Boland, Yu, Sullivan-Bolyai y Tamborlane, 1998; Ingersoll y Marrero, 1991).

Basándose en la literatura disponible sobre el tema, Delamater y colaboradores (2001) afirman que los pacientes con diabetes mellitus presentan una mayor prevalencia de trastornos psiquiátricos que los sujetos de la población general. En este sentido, Jacobson (1996) ha encontrado en adultos insulino-dependientes una mayor comorbilidad con trastornos afectivos y de ansiedad. Teniendo en cuenta esto, algunos autores han pretendido analizar el impacto de la psicopatología en la calidad de vida de las personas con diabetes mellitus. La mayoría de ellos han encontrado que altos niveles de sintomatología depresiva o la presencia de un trastorno depresivo disminuye la calidad de vida de adultos con ambos tipos de diabetes (Jacobson, Groot y Samson, 1997; Peyrot y Rubin, 1997, 1999; Wändell, 2005) asociándose con un peor control glucémico (Berlin et al., 1997; Lustman, Griffith y Clouse, 1997; Lustman et al., 2000; McGrady y Horner, 1999; Lloyd, Dyert y Barnett, 2000) y con complicaciones de la salud (Aalto et al., 1997; Peyrot y Rubin, 1997,1999). Por otro lado, también se ha encontrado que la calidad de vida de pacientes con DM1 disminuye cuando éstos experimentan altos niveles de ansiedad o padecen algún trastorno de este tipo (Jacobson et al., 1994). Además, al igual que en la depresión, unos niveles de ansiedad elevados también se relacionan con un pobre control glucémico en adultos con diabetes tipo 1 y 2 (Berlin et al., 1997; Lloyd et al., 2001; McGrady y Horner, 2001), así como con complicaciones de la salud (Peyrot y Rubin, 1997).

Otra área de estudio que se ha relacionado con la calidad de vida, ha sido el locus de control (LOC) relacionado con la salud, que en el caso concreto de la diabetes sería el lugar en el que la persona sitúa su capacidad de controlar los eventos que le ocurren relacionados con la enfermedad. Esta capacidad puede ubicarse en el propio sujeto (LOC interno) o fuera de él (LOC externo). En estudios realizados en adultos con ambos tipos de diabetes, se ha encontrado que un LOC interno se relaciona con una mayor calidad de vida (Aalto et al., 1997; Gillibrand y Stevenson, 2006; Keers et al., 2004), un mejor control glucémico (O'Hea et al., 2005; Reynaert et al., 1995; Stenström, Wikby, Andersson y Ryden, 1998) y una menor sintomatología depresiva y ansiosa (Peyrot y Rubin, 1994) que los pacientes que tienen un LOC externo. Para explicar estos resultados se sugiere que los pacientes que tienen un LOC interno, al considerarse más responsables de sus resultados, perciben los beneficios de tener una buena adherencia al tratamiento (Aalto y Uutela, 1997) y presentan un mejor ajuste a la diabetes (Helgeson y Franzen, 1998) que los pacientes que tienen un LOC externo.

El objetivo de este estudio ha sido analizar si variables psicológicas (depresión, ansiedad y locus de control interno) y metabólicas (control glucémico e hipoglucemias graves) pueden predecir la calidad de vida de pacientes con DM1. 
Tras revisar el estado actual del tema, nuestra hipótesis se plantearía en los siguientes términos: "las variables psicológicas depresión, ansiedad y locus de control interno, así como las variables metabólicas control glucémico e hipoglucemias graves serán buenas predictoras de la calidad de vida de los pacientes con DM1".

\section{Método}

\section{Muestra}

Se seleccionaron 46 pacientes atendidos en la Unidad de Diabetes del Servicio de Endocrinología del Hospital Regional Universitario Carlos Haya de Málaga (España). Siguiendo las directrices de la declaración de Helsinki (Faden, Beauchamp y King, 1986) se obtuvo consentimiento informado de los pacientes que participaron en este estudio. Treinta y tres pacientes formaron parte finalmente de este estudio. Los criterios de inclusión fueron los siguientes: pacientes con diabetes tipo 1 en tratamiento intensivo multidosis con insulina glargina o NPH y análogos de acción rápida (Humalog), más de 2 años de evolución, mayores de 14 años y con un péptido C (PC) menor de 0,5 ng/ml. Como criterios de exclusión se consideraron la diabetes tipo 2, una diabetes tipo 1 en tratamiento convencional (una o dos inyecciones/día) y alteraciones psicológicas incapacitantes. Las características demográficas y clínicas de los pacientes se muestran en las tablas 1, 2 y 3 .

\section{Instrumentos de evaluación}

- Diabetes Quality of Life (DQOL) (Diabetes Control and Complications Trial Research
Group, 1988). Se utilizó una versión adaptada a la población española de este cuestionario (Millán, Reviriego y del Campo, 2002). Este instrumento mide la calidad de vida de personas con diabetes. Está compuesto por 43 ítems que forman 4 dimensiones: "Satisfacción con el tratamiento" (15 ítems), "Impacto del tratamiento" (17 ítems), "Preocupación social/ vocacional" (7 ítems) y "Preocupación relativa a los efectos futuros de la diabetes" (4 ítems). Cada ítem tiene 5 opciones de respuesta de tipo Likert que puntúan del 1 al 5. En la subescala de satisfacción las respuestas a cada ítem oscilan entre "muy satisfecho" (1 punto) a "nada satisfecho" (5 puntos). En las otras tres subescalas el rango de las respuestas es el siguiente: "nunca" (1 punto) a "siempre" (5 puntos). Puede obtenerse una puntuación total y una puntuación por subescalas. Además, hay que tener en cuenta que una menor puntuación implica una mejor calidad de vida. Está diseñado para ser autoadministrado. Los datos de fiabilidad y validez del cuestionario referidos por sus autores son adecuados. En nuestro estudio hemos encontrado, al igual que los autores de la escala original, una adecuada consistencia interna tanto en las subescalas como en el total del DQOL (DQOL-Satisfacción con el tratamiento, $\alpha=0.68$; DQOL-Impacto del tratamiento $\alpha=0.87$; DQOL-Preocupación por aspectos sociales-vocacionales $\alpha=0.70$; DQOL-Preocupación por efectos futuros diabetes $\alpha=0.59$; DQOL-Total, $\alpha=0.89$ ).

- Inventario de Depresión de Beck (BDI) (Beck, Ward, Mendelson, Mock y Erbaugh, 1961). Se utilizó una versión adaptada a la población española de este cuestionario (Sanz, Perdigón y Vázquez, 2003). Está diseñado para evaluar la

Tabla 1. Características demográficas y clínicas de la muestra utilizada en el estudio

$\mathrm{n}$

Edad (años)

Duración de la diabetes (años)

$\operatorname{HbA1c}(\%)$

Hipoglucemias graves (últimos seis meses)

\section{3}

$31.09 \pm 11.16$

$14.82 \pm 7.35$

$8.62 \pm 1.73$

$0.72 \pm 1.12$ 
Tabla 2. Características demográficas de la muestra utilizada en el estudio

\begin{tabular}{|c|c|c|}
\hline & $\mathrm{n}$ & $\%$ \\
\hline \multicolumn{3}{|l|}{ Sexo } \\
\hline Hombres & 13 & 20 \\
\hline Mujeres & 39.4 & 60.6 \\
\hline \multicolumn{3}{|l|}{ Ocupación } \\
\hline Empleado & 19 & 57.6 \\
\hline Desempleado & 3 & 9.1 \\
\hline Jubilado & 0 & 0 \\
\hline De baja & 0 & 0 \\
\hline Ama/o de casa & 3 & 9.1 \\
\hline Estudiante & 8 & 24.2 \\
\hline \multicolumn{3}{|l|}{ Estado civil } \\
\hline Soltero/a & 17 & 51.1 \\
\hline Casado/a & 11 & 33.3 \\
\hline Separado/a & 2 & 6.1 \\
\hline Divorciado/a & 1 & 3.0 \\
\hline Viudo/a & 1 & 3.0 \\
\hline Pareja de hecho & 1 & 3.0 \\
\hline \multicolumn{3}{|l|}{ Estudios realizados } \\
\hline Conocimientos lectoescritura & 0 & 0 \\
\hline Estudios primarios & 11 & 33.3 \\
\hline Estudios de bachiller & 8 & 24.2 \\
\hline Estudios de FP & 1 & 3.0 \\
\hline Diplomado & 5 & 15.2 \\
\hline Licenciado & 8 & 24.2 \\
\hline
\end{tabular}

Tabla 3. Complicaciones crónicas de la diabetes en la muestra utilizada en el estudio

\begin{tabular}{lcccc}
\hline Complicaciones crónicas & SI & & NO \\
\hline & $\mathrm{n}$ & $\%$ & $\mathrm{n}$ & $\%$ \\
\hline Retinopatía preproliferativa & 5 & 15.2 & 25 & 75.8 \\
Nefropatía & 0 & 0 & 33 & 100 \\
Polineuropatía & 1 & 3 & 29 & 87.9 \\
\hline
\end{tabular}

intensidad de la sintomatología depresiva que presenta un individuo. El instrumento es autoaplicable y consta de 21 preguntas, cada una de ellas con cuatro afirmaciones que describen el espectro de severidad de la categoría sintomática y conductual evaluada. En todos los casos, el primer enunciado tiene un valor de 0 , que indica la ausencia del síntoma, el segundo de un punto, el tercero de dos puntos y el cuarto de 3 puntos, que constituye la severidad máxima del síntoma. El instrumento original y sus adaptaciones al castellano han mostrado validez y fiabilidad adecuadas para su empleo en el ejercicio clínico y de la investigación (Ramos, 1986; Torres, Hernández y Ortega, 1991). El BDI ha demostrado ser un instrumento efectivo para detectar depresión en personas con diabetes y otras enfermedades crónicas (Lustman, Griffith y Clouse, 1997). En nuestro estudio, el instrumento ha demostrado una adecuada consistencia interna $(\alpha=0.89)$.

- Cuestionario de Ansiedad Estado/Rasgo (STAI) (Spielberger, Gorsuch y Lushene, 1968). Se utilizó la adaptación al castellano realizada por Seisdedos (1988). Este cuestionario mide la ansiedad como estado y como rasgo y consta de 40 preguntas, las primeras veinte están dirigidas a detectar sintomatología ansiosa como reacción transitoria (subescala de ansiedad estado, STAI-E) y las veinte siguientes buscan la presencia de sintomatología ansiosa como rasgos persistentes de ansiedad (subescala ansiedad rasgo, STAI-R). Las preguntas del STAI se contestan en una escala tipo Likert que puntúa de 0 a 3 . En la subescala de ansiedad estado las respuestas de cada ítem oscilan entre "nada" (0 puntos) y "mucho" (3 puntos). En la subescala de ansiedad rasgo el 
rango de las respuestas de cada ítem es el siguiente: "casi nunca" (0 puntos) hasta "casi siempre" (3 puntos). El STAI posee validez discriminativa y una buena consistencia interna. En nuestro estudio hemos encontrado una excelente consistencia interna en ambas subescalas (STAIE, $\alpha=0,90$; STAI-R, $\alpha=0,88$ ).

- Escala de Lugar de Control (MHLC) de Wallston, y de Strudler (1981) que evalúa donde sitúa la persona el lugar de control o locus de control (LOC) de su salud, es decir, si la persona piensa que por sí misma puede vencer el obstáculo de salud o al menos controlarlo (LOC interno) o piensa que no puede controlar las variables de la salud y que su evolución o afrontamiento de un hecho negativo depende de variables externas como la suerte, el personal sanitario, la medicación, etc. (LOC externo). Este autoinforme está compuesto de 18 ítems de los cuales 6 valoran un LOC interno, y 12 evalúan un LOC externo. Sus preguntas se contestan en una escala tipo Likert ( $1=$ completamente en desacuerdo, a $6=$ completamente de acuerdo). Los autores del instrumento informan de fiabilidad y validez adecuadas para su empleo en el ejercicio clínico y de la investigación. En nuestro estudio, la consistencia interna encontrada es aceptable $(\alpha=0,45)$.

- Análisis de sangre: la evaluación objetiva del control glucémico se realizó mediante la hemoglobina glicosilada (HbA1c) que se midió a través de una cromatografía líquida de alta presión (HPLC) con un aparato modelo Kyoto Daiichi Kageki. La HbA1c cuantifica los valores de glucemia del paciente en los últimos tres meses aproximadamente. Basándonos en las recomendaciones sobre control glucémico para adultos con diabetes de la Asociación Americana de Diabetes (ADA, 2007), se define un control glucémico bueno cuando la HbA1c es inferior al $7 \%$.

- Historias clínicas: se utilizaron para recoger las hipoglucemias graves producidas en los últimos 6 meses. La Asociación Americana de Diabetes (ADA, 2005) define la hipoglucemia como el episodio clínico en el que concurren una medición de glucemia plasmática menor de
$70 \mathrm{mg} / \mathrm{dl}$ y una sintomatología típica (temblor, palpitaciones, sudoración, sensación de hambre, parestesias, etc.). La ADA (2005) también define la hipoglucemia grave como un acontecimiento en el que se producen síntomas neuroglucopénicos (sensación de calor, debilidad, confusión, cambios en el comportamiento, convulsiones, coma o muerte) que son resultado de la deprivación cerebral de glucosa y en el que se requiere la asistencia de otra persona para ayudar a revertir el cuadro clínico independientemente de que ocurra pérdida de conciencia o no. Según la ADA (2005) puede considerarse una reducción significativa de las hipoglucemias graves cualquier disminución (10-20 \%) de dichos episodios.

\section{Procedimiento}

El estudio se llevó a cabo íntegramente en una consulta perteneciente a la Unidad de Diabetes del Servicio de Endocrinología del Hospital Regional Universitario Carlos Haya de Málaga (España). En primer lugar, se recogió información sobre las variables sociodemográficas (edad, estudios, ocupación, etc.) y clínicas (hipoglucemias graves en los últimos seis meses, complicaciones crónicas de la diabetes, etc.). En segundo lugar, se registraron las medidas antropométricas de los pacientes (peso, talla, cintura/cadera) y se realizaron extracciones de sangre para posteriormente analizar las muestras. Posteriormente, se llevó a cabo una evaluación psicológica mediante la administración de una batería de tests en formato de entrevista (estructurada) con el fin de recoger datos sobre las variables psicológicas (depresión, ansiedad y locus de control) y de calidad de vida de los pacientes. Los tests se aplicaron con el siguiente orden de presentación: BDI, DQOL, MHLC y STAI.

Para contrastar la hipótesis se realizó un análisis de regresión múltiple con todos los datos de las variables objeto de estudio utilizando la versión 12 del programa estadístico SPSS para Windows. En él la calidad de vida (evaluada con el DQOL) ha constituido la variable dependiente $\mathrm{y}$, como variables predictoras se consideran, la depresión, la ansiedad, 
el locus de control interno, el control glucémico y las hipoglucemias graves, medidas mediante el BDI, el STAI, el MHLC, la hemoglobina glicosilada (HbA1c) y los datos de las historias clínicas respectivamente.

\section{Resultados}

El conjunto de las variables predictoras explican algo más de la mitad de la varianza de la variable calidad de vida $\left(\mathrm{R}^{2}=0,593\right)$. Como puede observarse en la tabla 4, la depresión (Beta $=0,525 ; \mathrm{t}=3,321$; $\mathrm{p}=0,002)$ y la ansiedad-rasgo $($ Beta $=0,595 ; \mathrm{t}=4,058$; $\mathrm{p}=0,000)$ son buenos predictores de la calidad de vida de los pacientes con DM1, de manera que una alta puntuación en depresión o en ansiedad-rasgo predice una alta puntuación en el DQOL y, por tanto, una peor calidad de vida en estos pacientes. El resto de las variables predictoras (ansiedad-estado, LOC interno, hemoglobina glicosilada e hipoglucemias graves) no alcanzan la significación estadística. Por tanto, la hipótesis que habíamos planteado se cumple parcialmente. trol glucémico e hipoglucemias graves) no se relacionaron con la calidad de vida de los pacientes con esta enfermedad.

Al comparar estos resultados con los hallazgos de los estudios que han analizado el impacto del estado emocional (depresión y ansiedad) en la calidad de vida de pacientes con ambos tipos de diabetes (Jacobson et al., 1997; Peyrot y Rubin, 1997, 1999; Wändell, 2005), comprobamos que, nuestros resultados coinciden con los de estos estudios. Así, al igual que en dichos estudios, hemos encontrado que altos niveles de depresión y ansiedad (rasgo) se relacionan con una disminución de la calidad de vida de los pacientes con DM1. Aunque, la ansiedad-estado no tuvo ningún efecto significativo sobre la calidad de vida de estos pacientes.

La mayoría de los estudios refieren que el control glucémico de adultos con diabetes mellitus tipo $1 \mathrm{se}$ relaciona con su calidad de vida (Tan et al., 2005; Weinger y Jacobson, 2001; Wikby et al., 1993). Sin embargo, al igual que en el presente estudio, algunos autores no han relacionado la calidad de vida con la hemoglobina glicosilada en pacientes con DM1 (Grey et al., 1998; Ingersoll y Marrero, 1991).

Tabla 4. Predictores de la variable dependiente "calidad de vida"

\begin{tabular}{lccc}
\hline Predictores de calidad de vida (DQOL) & Beta & $\mathrm{t}$ & Sig. \\
\hline Depresión (BDI) & 0.525 & 3.321 & 0.002 \\
Ansiedad estado (STAI-E) & 0.256 & 1.404 & 0.171 \\
Ansiedad-rasgo (STAI-R) & 0.595 & -0.992 & 0.000 \\
LOC interno (MHLC) & -0.178 & -0.578 \\
Hemoglobina glicosilada (HbA1c) & -0.113 & 0.329 \\
Hipoglucemias graves (episodios) & 0.114 & 0.512 \\
\hline
\end{tabular}

\section{Discusión}

En este estudio nos planteamos analizar si algunas variables psicológicas (depresión, ansiedad y locus de control interno) y metabólicas (control glucémico e hipoglucemias graves) podían predecir la calidad de vida de pacientes con DM1. Hemos encontrado que la sintomatología depresiva y ansiosa (rasgo) de los pacientes con DM1 puede explicar la calidad de vida de dichos pacientes. Sin embargo, el resto de variables (locus de control interno, con-
Existe cierto consenso sobre la relación entre la presencia de complicaciones médicas de la enfermedad y una disminución de la calidad de vida de adultos con DM1 (Aalto et al., 1997; Hahl et al., 2002). Nuestros resultados no apoyan estos hallazgos, ya que hemos encontrado que las hipoglucemias graves no se relacionan con la calidad de vida percibida de estos pacientes.

En cuanto a la percepción de control, la literatura especializada en este tema sugiere que el locus de control interno de los pacientes con DM1 se asocia 
con una mejor calidad de vida (Aalto et al., 1997; Gillibrand y Stevenson, 2006; Keers et al., 2004). Nuestros resultados no apoyarían a este grupo de investigaciones, ya que no hemos encontrado que la calidad de vida se relacione con un locus de control interno en los pacientes con DM1, por tanto, podríamos decir que el hecho de que estos pacientes se impliquen más en el cuidado de su enfermedad y se consideren más responsables de los resultados del mismo (locus de control interno) no pueden explicar adecuadamente la percepción de calidad de vida del paciente.

En suma, hemos encontrado que la ansiedad (rasgo) y la depresión son dos importantes variables que explican la calidad de vida de los pacientes con
DM1. Así, al igual que otros autores han demostrado que las variables fisiológicas o metabólicas pueden ser buenos predictores de calidad de vida en personas con esta enfermedad (Aalto et al., 1997; Hahl et al., 2002; Tan et al., 2005; Weinger y Jacobson, 2001), los resultados de este estudio evidencian que las variables psicológicas también pueden serlo. Por tanto, según nuestros resultados, para mejorar la calidad de vida de estos pacientes habría que promover tanto la prevención como un adecuado tratamiento psicológico de la sintomatología depresiva y ansiosa de los pacientes con diabetes. Es por esto, por lo que se hace necesaria la presencia de la figura del psicólogo dentro de los equipos multidisciplinares que atienden a las personas con esta enfermedad crónica.

\section{Extended Summary}

\section{Introduction}

Quality of life is a multidimensional construct that includes three dimensions: physical, psychological and social. The physical dimension refers to the perception of physical or health condition (absence of disease), the symptoms produced by disease, and adverse effects of treatment. The psychological dimension indicates an individual's perception of his/her cognitive and emotional state such as fear, anxiety, isolation, loss of self esteem, uncertainty about the future, and so on. It also includes personal beliefs, both spiritual and religious, such as the meaning of life and attitude to suffering. Finally, the social dimension refers to the individual's perception of interpersonal relationships and social roles in life and the need for family and social support, doctor-patient relationship and job performance.

Its use has spread to many fields, including health. Thus the term "Health-Related Quality of Life" (HRQOL) was born. It expresses the fact that "the different aspects of a person's life are strongly affected by changes in health status "(Schalock \& Verdugo, 2003) and "the effects of the disease and treatment on the physical, emotional and social well-being"(Cella et al., 1999).

Although research on the quality of life in patients with diabetes mellitus is fairly recent, there is abundant literature about adults with both types of diabetes, which has been found a lower quality of life in these patients than in the general population, particularly in relation to physical functioning and well-being (Rubin \& Peyrot, 1999).

Multiple diabetes-related factors have been reported that can affect quality of life of patients with this disease (Anarte, 2004): the specific impact of the diagnosis; treatment demands which can put the person with diabetes or the family in situations that often engender the emergence of negative emotional reactions; uncertainty about possible future complications; fear of hypoglycaemia and its negative consequences (physical, cognitive, motor, etc.); frustration with inexplicably high or low levels glucose; and carrying out your daily medical regime, which imposes restrictions on the family lifestyle (injections, testing, meal planning, exercise, and so on).

The quality of life of adult patients with diabetes mellitus has been linked to complications of the disease (Rubin and Pierrot, 1999). Thus, as the number and severity of symptoms of medical complications increases, the quality of life reduces significantly (Aalto, Uutela and Aro, 1997; Hahl et al., 2002). These findings have also been found (Ambler et al, 2006) in Australian children and adolescents with type 1 diabetes mellitus (DM1).

Empirical evidence has been found about a relationship between glycemic control of patients with diabetes mellitus and quality of life (Rubin and Pierrot, 
1999), so that, by increasing patient's glycemic control, it also enhances the quality of life. However, there are also studies that have found no relationship.

On the other hand, patients with diabetes mellitus have a higher prevalence of psychiatric disorders than subjects in the general population (Delamater et al, 2001), having greater comorbidity with mood and anxiety disorders (Jacobson, 1996). Thus, a high level of depressive symptoms or the presence of a depressive disorder and a high level of anxiety diminishes the quality of life of adults with both types of diabetes (Jacobson et al., 1997; Peyrot and Rubin, 1997, 1999) when associated with poorer glycemic control (Lustman, Griffith, and Clouse, 1997, Lustman et al., 2000; McGrady and Horner, 1999) and with health complications (Peyrot and Rubin, 1997,1999).

Another area of study that has been linked to quality of life, has been the locus of control (LOC) related to health, which in the case of diabetes would be the place where the person puts his/her ability to control events that occur relating to the disease. This capability can be located either in the subject (internal LOC) or outside (external LOC). In studies in adults with both types of diabetes, it has been found that an internal LOC is associated with a higher quality of life (Gillibrand and Stevenson, 2006), better glycemic control (O'Hea et al., 2005), and fewer depressive symptoms or anxiety (Peyrot and Rubin, 1994) than patients who have external LOC.

The aim of this study was to examine whether psychological variables (depression, anxiety and locus of control) and metabolic variables (glycemic control and severe hypoglycaemia) can predict the quality of life of patients with DM1.

After reviewing the current status of this topic, our hypothesis is the following: "psychological variables (depression, anxiety and locus of control) and metabolic variables (glycemic control and severe hypoglycaemia) are good predictors of the quality of life DM1 patients".

\section{Method}

\section{Sample}

We selected 46 patients treated at the Diabetes
Unit of the Endocrinology Service at the "Hospital Regional Universitario Carlos Haya" in Malaga (Spain). Following the guidelines of the Declaration of Helsinki (Faden, Beauchamp and King, 1986) informed consent was obtained from 33 patients who participated in this study. Inclusion criteria were: patients with DM1 in multidose intensive treatment with insulin glargine or NPH and rapidacting analogues (Humalog), more than 2 years of evolution, over 14 years with a peptide C (PC) of less than $0.5 \mathrm{ng} / \mathrm{ml}$. Exclusion criteria were considered type 2 diabetes, type 1 diabetes therapy (one or two injections / day), incapacitating psychological disturbances. The demographic and clinical characteristics of patients are shown in Tables 1,2 and 3 .

\section{Measures}

- Diabetes Quality of Life (DQOL) (Diabetes Control and Complications Trial Research Group, 1988). We used a version of this questionnaire adapted to the Spanish population (Millan, Reviriego and Field, 2002). This instrument specifically evaluates the quality of life in patients with diabetes.

- Beck Depression Inventory (BDI) (Beck, Ward, Mendelson, Mock and Erbaugh, 1961). We used a version of this questionnaire adapted to the Spanish population (Sanz, Perdigon and Vazquez, 2003). It is designed to evaluate the intensity of depressive symptoms that presents an individual.

- State-Trait Anxiety Inventory (STAI) (Spielberger, Gorsuch and Lushene, 1968). Adaptation by Seisdedos (1988) was used. This questionnaire measures anxiety as a state and as a trait.

- Health locus of Control Scale (MHLC) of Wallston, and Strudler (1981) which assesses where the person locates control over his/her health. That is, whether the patients think they themselves can overcome their health problems, or at least control them (internal LOC), or if they think that they cannot control the health variables and their evolution, or if negative coping depends on external variables such 
as luck, health workers, medication, and so on. (external LOC).

- Blood tests: objective assessment of glycemic control performed by glycosylated hemoglobin (HbA1c) was measured through a high pressure liquid chromatography with a model apparatus Kageki Kyoto Daiichi. HbA1c quantifies the patient's blood glucose levels in the last three months. It was based on the recommendations about glycemic control for adults with diabetes from the American Diabetes Association (ADA, 2007) which defines good glycemic control as when the HbA1c is less than $7 \%$.

- Patient histories were used to collect the produced severe hypoglycaemia in the last 6 months. Hypoglycaemia (The American Diabetes Association, ADA, 2005) is a clinical event in which the measurement of plasma glucose below $70 \mathrm{mg} / \mathrm{dl}$ concurs with typical symptoms (tremor, palpitations, sweating, hunger, paresthesias, and so on). Hypoglycaemia is severe when the patient has symptoms neuroglycopenic (warm sensation, weakness, confusion, behavioral changes, convulsions, coma or death) that result from cerebral glucose deprivation and requires the assistance of another person to reverse the clinical picture, regardless of whether loss of consciousness occurs or not (ADA, 2005).

\section{Procedure}

The study was carried out entirely in tests at the Diabetes Unit of Endocrinology Service (Hospital Regional Universitario Carlos Haya in Malaga. Spain). First, information was gathered on sociodemographic variables (age, education, occupation, etc..) and clinical variables (severe hypoglycemia in the past six months, chronic complications of diabetes, etc.). Second, anthropometric measurements were recorded for patients (weight, height, waist / hip), and blood samples were performed for further analysis. Subsequently, a psychological evaluation was conducted by administering a battery of tests in interview format (structured) in order to collect data on psychological variables (depression, anxiety and locus of control) and quality of life in patients. The tests were applied in the following order of presentation: BDI, DQOL, MHLC and STAI.

To test the hypothesis a multiple regression analysis was performed using version 12 of SPSS software for Windows. Quality of life (assessed with DQOL) was the dependent variable and the predictor variables were depression, anxiety, internal locus of control, glycemic control and severe hypoglycaemia.

\section{Results}

The set of predictor variables explain just over half of the variance in the quality of life variable ( 2 $=0.593$ ). As shown in Table 4, depression (Beta $=$ $0.525, \mathrm{t}=3.321, \mathrm{P}=0.002)$ and trait anxiety (Beta $=$ $0.595, \mathrm{t}=4.058, \mathrm{P}=0.000)$ are good predictors of quality of life in DM1 patients, so high scores on depression or trait anxiety predict a high score on the DQOL and therefore a lesser quality of life in these patients. The rest of the predictors (anxietystate, internal LOC, glycated hemoglobin and severe hypoglycemia) do not reach statistical significance. Therefore, this study did not find some results referred to in the scientific literature.

\section{Discussion}

In this study we considered examining whether some psychological variables (depression, anxiety and locus of control) and metabolic (glycemic control and severe hypoglycaemia) could predict the quality of life of patients with DM1. We have found that depressive symptoms, and anxiety (trait) of patients with DM1 may explain the quality of life for these patients. However, other variables (internal locus of control, glycemic control and severe hypoglycaemia) were not related to the quality of life of patients with this disease.

In summary, we found that anxiety (trait) and depression are two important variables that explain the quality of life of patients with DM1. So, like other authors who have shown that physiological or metabolic variables may be good predictors of qual- 
ity of life in people with this disease (Aalto et al., 1997; Hahl et al., 2002, Tan et al., 2005; Weinger and Jacobson, 2001), the results of this study show that psychological variables can also be good predictors in this respect. Therefore, according to our results, to improve the quality of life of these patients both prevention and proper psychological treatment of depressive and anxious symptoms of patients with diabetes would be important. For these reasons, the presence of the psychologist in multidisciplinary teams that care for people with chronic disease would also be necessary.

\section{Referencias}

Aalto, A.M. y Uutela, A. (1997). Glycemic control, self-care behaviors, and psychological factors among insulin treated diabetics: A test of an Extended Health Belief Model. International Journal of Behavioral Medicine, 4, 191-214.

Aalto, A.M., Uutela, A. y Aro, A.R. (1997). Health related quality of life among insulin- dependent diabetics: disease-related and psychosocial correlates. Patient Education and Counseling, 30, 215225.

Anarte, M.T. (2004). Importancia del estrés en la diabetes. Célula Beta, 18, 18-21.

Ambler, G.R., Fairchild, J., Craig, M.E. y Cameron, F.J. (2007). Contemporary Australian outcomes in childhood and adolescence type 1 diabetes: 10 years post the Diabetes Control and Complications Trial. Journal of Paediatrics and Child Health, 43, 403-410.

American Diabetes Association (2005). Committee Reports and Consensus Statements. Workgroup on hypoglycemia: defining and reporting hypoglycaemia in diabetes: a report of the American Diabetes Association Workgroup on Hypoglycemia. Diabetes Care, 28, 1245-49.

American Diabetes Association (2007). Standards of Medical Care in Diabetes-2007. Diabetes Care, 30, 4-41.

Beck, A.T., Ward, C.H., Mendelson, M., Mock, J. y Erbaugh, J (1961). An inventory for measuring depression. Archives of General Psychiatry, 4, 561-571.
Berlin, I., Bisserbe, J.C., Eiber, R., Balssa, N., Sachon, C., Bosquet, F. y Grimaldi, A. (1997). Phobic symptoms, particularly the fear of blood and injury, are associated with poor glycemic control in type 1 diabetic adults. Diabetes Care, 20, 176- 8 .

Cella, D.F., Diennen, K., Arnason, B., Reder, A., Webste, M., Karabastos, B., Chang, C., Lloyd, S., Mo, F, Stewart, J. y Stefoski, D. (1999). Validation of the functional assessment of multiple sclerosis quality of life instrument. American Academy of Neurology, 47, 129-139.

DeGroot, M., Jacobson, A.M., Samson, J.A. y Welch, G. (1999). Glycemic control and major depression in type 1 and type 2 diabetes mellitus. Journal of Psychosomatic Research, 46, 425435.

Delamater, A., Jacobson, A.M., Barbara, A., Daniel, C., Fisher, L., Lustman, P., Rubin, R. y Wisocki, T. (2001). Diabetes Care, 24, 1286-1292.

Diabetes Control and Complications Trial Research Group (1988). Reliability and validity of a Diabetes Quality-of-Life Measure (DQOL) for the Diabetes Control and Complications Trial (DCCT). Diabetes Care, 11, 725-32.

Diener, E. (1984). Subjective well-being. Psychological Bulletin, 95, 542-575.

Faden, R., Beauchamp, T. y King, N. (1986). A history and theory of informed consent. New York: Oxford University Press.

Fierro, A. (2006). Bienestar personal, adaptación social y factores de personalidad: Estudios con las Escalas Eudemon. Clínica y Salud, 17, 297318.

Gillibrand, R. y Stevenson, J. (2006). The extended health belief model applied to the experience of diabetes in young people. British Journal of Health Psychology, 11, 155-169.

Grey, M., Boland, E.A. yu, C., Sullivan-Bolyai, S. y Tamborlane, W.V. (1998). Personal and family factors associated with quality of life in adolescents with diabetes. Diabetes Care, 21, 909914.

Guttmann-Bauman, I., Flaherty, B.P., Strugger, M. y McEvoy, R.C. (1998). Metabolic control and quality-of-life self-assessment in adolescents with IDDM. Diabetes Care, 21, 915-918. 
Hahl, J., Hämäläinen, H, Sintonen, H., Simell, T., Arinen, S. y Simell, O. (2002). Health-related quality of life in type 1 diabetes without or with symptoms of long-term complications. Quality of Life Research, 11, 427-436.

Helgeson, V.S. y Franzen, P.L. (1998). The role of locus of control in adjustment in diabetes. Anxiety, Stress and Coping, 11, 113-136.

Ingersoll, G.M. y Marrero, D.G. (1991). A modified quality-of-life measure for youths: Psychometric properties. Diabetes Educator, 17, 114-118.

Jacobson, A.M. (1996). The psychological care of patients with insulin-dependent diabetes mellitus. New England Journal of Medicine, 334, 1249-1253.

Jacobson, A.M., de Groot, M. y Samson, J. (1994). Quality of life in patients with Type I and Type II diabetes mellitus. Diabetes Care, 17, 167-274.

Keers, J.C., Blaauwwiekel, E.E., Hania, M., Bouma, J., Scholten-Jaegers, S.M., Sanderman, R. y Links, T.P. (2004). Diabetes rehabilitation: development and first results of a Multidisciplinary Intensive Education Program for patients with prolonged self-management difficulties. Patient Education and Counseling, 52, 151-157.

Lloyd, C.E., Dyert, P.H. y Barnett, A.H. (2000). Prevalence of symptoms of depression and anxiety in a diabetes clinic population. Diabetic Medicine, 17, 198- 202.

Lustman, P.J., Griffith, L.S. y Clouse R.E. (1997). Depression in adults with diabetes. Seminary Clinical Neuropsychiatry, 2, 15-23.

Lustman, P.J., Anderson, R., Freedland, K, De Groot, M, Carney, R. y Clouse, R. (2000). Depression and poor glycemic control: a metaanalytic review of the literature. Diabetes Care, 23, $934-942$.

McGrady, A. y Horner, J. (2001). Role of mood in outcome of biofeedback assisted relaxation therapy in insulin dependent mellitus. Applied Psychophysiology Biofeedback, 24, 79- 88.

Millán, M.M., Reviriego, J. y del Campo, J. (2002):

Reevaluación de la versión española del Cuestionario Diabetes Quality of Life (EsDQOL). Endocrinología y Nutrición, 49, 322324.

Montero, I. y León, O.G. (2005). Sistema de clasifi- cación del método en los informes de investigación en Psicología. International Journal of Clinical and Health Psychology, 5, 115-127.

O’Hea, E., Grothe, K.B., Bodenlos, J.S., Boudreaux, E.D., White, M.A. y Brantley, P.J. (2005). Predicting medical regimen adherente: The interactions of Health Locus of Control Beliefs. Journal of Health Psychology, 10, 705-717.

Peyrot, M. y Rubin, R.R.. (1994). Structure and correlates of diabetes specific locus of control. Diabetes Care, 17, 994-1001.

Peyrot, M. y Rubin, R.R. (1997). Levels and risks of depression and anxiety symptomatology among diabetic adults. Diabetes Care, 20, 585590.

Peyrot, M. y Rubin, R.R (1999). Persistence of depression in diabetic adults. Diabetes Care, 22, 448-452.

Ramos, J. A. (1986). La validez predictiva del Inventario para la Depresión de Beck en castellano. Actas Luso-Españolas de NeurologíaPsiquiatría y Ciencias Afines, 14, 47-50.

Reynaert, C., Janne, P., Donckier, J., Buysschaert, M., Zdanowicz, N., Lejeune, D. y Cassier, L. (1995). LOC and metabolic control. Diabetes Metabolism, 21, 180-187.

Rubin R.R. y Peyrot M. (1999). Quality of life and diabetes. Diabetes Metabolism Research and Reviews, 15, 205-218.

Sanz, J., Perdigón, A.L. y Vázquez, C. (2003). Adaptación española del Inventario para la Depresión de Beck-II (BDI-II): 2. Propiedades psicométricas en población general. Clínica y Salud, 14, 249-280.

Schalock, R.L. y Verdugo, M.A. (2003). Calidad de vida. Manual para profesionales de la educación, salud y servicios sociales.Madrid: Alianza Editorial.

Seisdedos, N. (1988). Cuestionario de ansiedad estado-rasgo. Adaptación española. Madrid: TEA.

Spielberger C.D., Gorsuch R.L. y Lushene R.E. (1968). Manual for the State-Trait Anxiety Inventory (Self Evaluation Questionnaire). Palo Alto, California: Consulting Psychologists Press, Inc.

Stenström, U., Wikby, A., Andersson, P. O. y Ryden, 
O. (1998). Relationship between locus of control beliefs and metabolic control in insulin dependent diabetes mellitus. British Journal of Health Psychology, 3, 15-25.

Tan, S.M.K., Shafiee, Z., Wu, L.L. y Rey, J.M (2005). Factors associated with control of type I diabetes in Malaysian adolescents and young adults. International Journal of Psychiatry in Medicine, 35, 123-136.

Torres, A., Hernández, E. y Ortega, H. (1991). Validez y reproducibilidad del Inventario para Depresión de Beck en un hospital de cardiología. Salud Mental, 14, 1-6.

Wallston, K.A., Stein, M.J. y Smith, C.A. (1994). Form C of the MHLC scales: a condition - specific measure of locus of control. Journal of Personality, 63, 534-553.
Wallston, K.A. y Strudler, B. (1981). Health Locus of control scales. Research with the locus of control construct, 1, 189-243.

Wändell, P.E. (2005). Quality of life of patients with diabetes mellitus. Scandinavian Journal of Primary Health Care, 23, 68-74.

Weinger, K. y Jacobson, A.M. (2001). Psychosocial and quality of life correlates of glycemic control during intensive treatment of type 1 diabetes. Patient Education and Counseling, 42, 123-131.

Wikby, A., Hörnquist, J.A., Stenström, U. y Andersson, P.O. (1993). Back-ground factors, long term complications, quality of life and metabolic control in insulin dependent diabetes. Quality of Life Research, 2, 281-286.

World Health Organization (1994). Quality of life. Geneva: WHO.

Artículo recibido: 14/12/2009

Revisión recibida: 15/01/2010

Aceptado: 15/02/2010 